\title{
Nonlinear optical properties of Au-nanoparticles conjugated with lipoic acid in water
}

\author{
M. Trejo-Durán \\ mtrejo@ugto.mx \\ D. Cornejo-Monroy \\ E. Alvarado-Méndez
}

A. Olivares-Vargas

V. M. Castaño

\begin{abstract}
DEM-DICIS Universidad de Guanajuato, Carretera Salamanca-Valle de Santiago km 3.5 + 1.8 Comunidad de Palo Blanco, Salmanaca, Guanajuato 33885, México

Instituto de Ingeniería y Tecnología, Universidad Autónoma de Ciudad Juárez, Av. del Charro No. 450 Norte, Ciudad Juárez, Chihuahua 32310, México

Departamento de Electronica Universidad de Guanajuato, Carretera Salamanca-Valle de Santiago km 3.5 + 1.8 Comunidad de Palo Blanco, Salmanaca, Guanajuato 33885, México

Student at Departamento de Estudios Multidisciplinarios DICIS Universidad de Guanajuato, Carretera Salamanca-Valle de Santiago km 3.5 + 1.8 Comunidad de Palo Blanco, Salmanaca, Guanajuato 33885, México

Centro de Fisica Aplicada y Tecnologia Avanzada Universidad Nacional Autonoma de Mexico, Boulevard Juriquilla 3001 Quereetaro, Queretaro 76230, México
\end{abstract}

Gold nanoparticles were chemically conjugated with lipoic acid to control their optical properties. Z-scan and other optical techniques were used to characterize the non-linear behavior of the resulting nanostructured materials. The results show that the nonlinearity is of thermal origin, which can be controlled by the use of lipoic acid as well as other organic molecules conjugated onto metal nanoparticles. In particular, the presence of lipoic acid increases $n_{2}, \theta$ and $d n / d t$.

[DOI: http://dx.doi.org/10.2971/jeos.2014.14030]

Keywords: Nanoparticles, nonlinear optical properties, z-scan, lipoic acid

\section{INTRODUCTION}

Nowadays nanoparticles have reached great interest into various researching areas, due to unique electronic, magnetic and optical properties $[1,2]$. Some potential applications go from biological to electronic applications, some of them are: sensor technology [3]-[5], biological labeling, drug delivery system and treatment for some cancers [6, 7], surface-enhanced Raman scattering, paintings [8], optical diodes, high speed optical logic devices [9]; on the other hand, properties of nanoparticles as the thermal conductivity and nonlinear optical properties, are interesting, because by using nanoparticles it is possible to elevate the thermal conductivity of fluids being these metallic [10]-[12], or nonmetallic [13, 14] with different shapes and sizes, and suspended in different fluids, which modified the nonlinear optical properties of fluids. Nonlinear optical gives origin to nonlinear optical phenomena as modulation instability $[15,16]$, self-diffraction $[17,18]$, nonlinear shock waves $[19,20]$, stabilization of complex soliton structures [21, 22], and these phenomena can be applied to design optical limiters, multiplexors, modulators, ultrafast optical communication [23]-[25]. In particular, the behavior of Au-nanoparticles in different media has been reported by using the $\mathrm{z}$-scan technique [25]-[27]. However, optical properties of Au nanoparticles can be modified when they are conjugated with other molecules. This is interesting because it plays a crucial role in determining their linear and nonlinear optical properties [29]-[33]. In this work we study how the nonlinear optical properties of fluids with Au-nanoparticles present change. They were performed using the Z-scan technique (at millisecond range), and applying two models: Bahae formalism and thermal lens to obtain $n_{2}, d n / d t$. [23]-[33]

\section{THEORY}

Consider an incident Gaussian beam in a medium. The medium absorbs the light and then gives a local heating immediately, the temperature modified in the surroundings of a laser beam producing the refractive index changes too, the nonlocal effect and the influence in the nonlinear refractive index present, have to be considered. The refractive index is assumed to depend linearly on temperature and the nonlinearity is due to this nonlocal dependence of the refractive index on the local light intensity. The magnitude of this nonlocality can be estimated by degree of nonlocality. This parameter depends on the optical and thermal properties of the material and can be given by Ghofraniha et al [34]

$$
\sigma=\sqrt{\frac{\kappa\left|n_{2}\right|}{\alpha_{0}\left|\frac{\partial n}{\partial T}\right| \omega_{0}^{2}}}
$$

Where $\omega_{0}$ is the minimum beam waist, $n_{2}, \mathrm{k}, d n / d T, \alpha_{0}$ are the nonlinear refractive index, the heat conductivity, the thermooptical and the linear absorption coefficients of the medium. The larger the value of $\sigma$, the more nonlocal is the medium. 
On the other hand if $\sigma=0$ the medium can only present a local nonlinearity

The elements that can be measured are $\alpha_{0}, n_{2}$, and $d n / d T$, these last are nonlinear optical properties of the medium. One of the easiest techniques to study the nonlinear optical properties is the Z-scan technique. It was developed by Bahae [35] and it is the most known for determining the nonlinearity of materials. To interpret the results obtained by z-scan technique we used diverse models; the Sheik-Bahae formalism and the thermal lens model, [36] because both help to describe nonlocal systems in time; Bahae formalism does it once the system has reached a steady status (we used a chopper at milliseconds to achieve this), and the thermal model, which describes the nonlinearity with thermal origin.

\subsection{Sheik-Bahae Formalism}

Consider a medium illuminated by a Gaussian laser beam; it is an electric field $(\boldsymbol{E})$, and respectively frequency, the nonlinear refraction index $n_{2}$ can be defined by:

$$
n=n_{0}+\frac{1}{2} n_{2} E^{2}
$$

Where $n$ is the refractive index. For a sample where nonlinear absorption is negligible, the on-axis normalized transmittance can be obtained approximately by:

$$
T(z)=1+\Delta \Phi_{0} \frac{4 x}{\left(1+x^{2}\right)\left(9+x^{2}\right)}
$$

Where $\Delta \Phi_{0}$ is the on axis nonlinear phase shift at focus, $x$ is the dimensionless sample position, $x=\frac{z}{z_{0}}, z_{0}=\frac{\pi \omega^{2}}{\lambda}$ is the Rayleigh parameter of the Gaussian beam with waist $\omega_{0}$. The peak-valley difference $\Delta T$ is:

$$
\Delta T=0.406\left|\Delta \Phi_{0}\right|
$$

The nonlinear phase shift is given by

$$
\Delta \Phi_{0}=\frac{2 \pi d\left(1-e^{-\alpha L}\right)}{\lambda \alpha} n_{2} I_{0}
$$

Where $\alpha$ is the linear absorption coefficient, $L$ is the thickness of the sample, $I_{0}$ is the peak intensity at the focus and $\lambda$ is the wavelength of the laser beam. An advantage of this method is that we can obtain the magnitude and sign of nonlinear refractive index by using a close aperture and the nonlinear absorption coefficient by employing an open aperture in the detector.

The nonlinear absorption coefficient $(\beta)$ is related to the transmittance by [38]

$$
\Delta T(z) \approx-\frac{q_{0}}{2 \sqrt{2}} \frac{1}{\left[1+Z^{2} / Z_{0}^{1}\right.}
$$

where $q_{0}=I_{0} L_{e f f} \beta$.

\subsection{Thermal Lens Model}

When a Gaussian beam is propagated across the absorbent medium, a local heating immediately occurs. This change of temperature around the beam laser gives origin to a change in the refractive index of the medium, papers describing this phenomena are found [35, 39]. Analysis of Gordon et al, has predicted a parabolic approximation to the temperature field and an approximation to the optical effects due the temperature as the behavior of a simple lens. The time dependent expression [40] for the far-field on-axis transmittance is

$$
\begin{aligned}
T_{N} & =\frac{I(z, t)}{I(z, 0)} \\
& =\frac{1}{1+\left(\frac{\theta}{1+t_{c} / 2 t}\right)\left(\frac{2 x}{1+x^{2}}\right)+\left(\frac{\theta}{1+t_{c} / 2 t}\right)^{2}\left(\frac{1}{1+x^{2}}\right)}
\end{aligned}
$$

Where $x=\frac{z}{z_{0}}, z_{0}=\frac{\pi \omega^{2}}{\lambda}, t_{c}=\frac{\omega^{2}}{4 k / C_{p}} \quad t_{c}$ is thermal diffusion time, $k$ is the thermal conductivity and $C_{p}$ is the specific heat per volume at constant pressure, The on-axis phase shift is given by

$$
\theta=\frac{\alpha P L}{\lambda \kappa} \frac{\partial n}{\partial T}
$$

Where $P$ is the power of the Gaussian beam and $T$ is the temperature. To lower power the nonlinear phase shifts $\theta \ll 1$, then the second term of Eq. (7) in the denominator is neglected. When the steady state has been reached can be considering $t>>t_{c}$ then Eq. (7) is given by

$$
T_{N}=\frac{1}{1+(\theta)\left(\frac{2 x}{1+x^{2}}\right)}
$$

The z-scan curve then corresponds to Eq. (9).

\section{EXPERIMENTAL SETUP}

Gold nanoparticles stabilized with lipoic acid were prepared taking into account Brust's method [41] to prepare small gold nanoparticles, and the sulfhydryl groups' affinity with gold for forming covalent bonds. Briefly, sodium borohydride was added to a solution of tetrachloroauric acid in water. The molar ratio between sodium borohydride and tetrachloroauric acid is directly related with the final gold particle size. The samples were prepared using a molar ratio of 5:1 between $\mathrm{NaBH}_{4}$ and $\mathrm{HAuCl}_{4}$, respectively. Once the gold nanoparticles were synthesized, lipoic acid (LA) or reduced lipoic acid (DHLA) were used to stabilize the gold nanoparticle colloids. To determine the quantity of LA and DHLA to be used, the size and concentration of the colloids were estimated according to W. Haiss et al. method [42]. Based on these results, and the estimated area occupied by one molecule, LA and DHLA

\begin{tabular}{|l|l|}
\hline Name & Solution \\
\hline 1Au & Au nanoparticules in water \\
\hline 2AuDHLA & Au nanoparticules with reduced lipoic acid in water \\
\hline 3AuDHLANaCl & Au nanoparticules with reduced lipoic acid and sodium chloride in water \\
\hline 4AuLA & Au nanoparticules with lipoic acid in water \\
\hline 5AuLANaCl & Au nanoparticules with lipoic acid and sodium chloride in water \\
\hline
\end{tabular}

TABLE 1 Characteristics of gold colloid samples. 


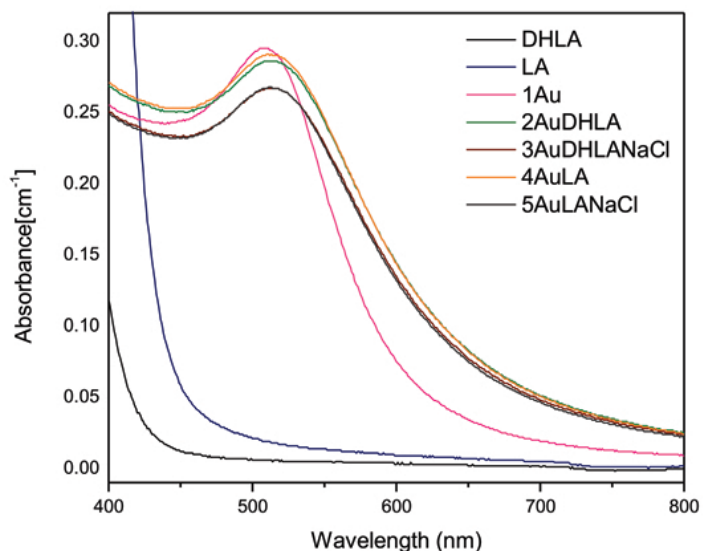

FIG. 1 UV-vis spectra of the different solutions.

plus thirty times more were calculated in order to ensure that all the surface of the gold nanoparticles were covered. Finally, $\mathrm{NaCl}$ was added to the samples to study the stability of gold colloids and the effect on the optical properties. Table 1 shows the different samples prepared. The concentration of nanoparticles in each solution was $300 \mathrm{nM} / 1$ (nanomols/litre).

The UV-vis spectra of the solutions are shown in Figure 1. Water as it is known, does not absorb in visible, note the lipoic acid and reduced lipoic acid do not absorb in visible too. The peak absorption band of Au nanoparticles suspended in water is at $508 \mathrm{~nm}$, with a valley at $450 \mathrm{~nm}$, which is characteristic of spherical gold nanoparticles. The solutions with lipoic acid and reduced acid lipoic do not affect the linear absorption but sodium chloride slightly decreases it. Since the Au nanoparticles have a diameter smaller than the wavelength of the incident light, surface plasmon resonance (SPR) takes place during our experiment. UV-vis shows a peak at $514 \mathrm{~nm}$, which corresponds precisely to a surface plasmon resonance absorption peak. This wavelength shift to the plasmon peak of $\mathrm{Au}$, appears when adding HLA, LA and NaCL. It is known that $\mathrm{Au}$ nanoparticles dispersed in solutions have great linear absorption and they absorb energy due to SPR of the electron. The absorbed energy will first be transfered to the phonon modes of the metal nanoparticles and then the surrounding liquid, so the local temperature will increase in the solution. Then, most of the nonlinear effects that we observe will have a thermal origin.

The z-scan experimental setup for the CW laser is shown schematically in Figure 2. CW Ar laser at $514 \mathrm{~nm}$ with variable power (using at 10, 16 and $25 \mathrm{~mW}$ ), propagating in $z$ direction, was focused using a lens (18 cm focus) and a waist beam of $29.16 \mu \mathrm{m}$ was obtained. A mechanical chopper was used to modulate the intensity, to measure at the millisecond range, providing nearly square pulses width of 20 and $10 \mathrm{~ms}$. The sample was moving using a control PC-system [43] to constant velocity. The liquid sample was collocated in a $2 \mathrm{~mm}$ thick cell, and moved along the z-direction; the transmitted intensity was measured through the finite aperture in the far field $(80 \mathrm{~cm})$ as a function of the sample position $z$; measured with respect to the focal plane. As the sample moves through the beam focus (at $z=0$ ), self-focusing or defocusing modifies

\begin{tabular}{c|c|c}
\hline Sample & $\begin{array}{c}\text { Peak } \\
(\mathbf{n m})\end{array}$ & $\begin{array}{c}\alpha \\
\left(\mathbf{c m}^{-\mathbf{1}}\right)\end{array}$ \\
\hline DHLA & 514 & 0.005 \\
LA & 514 & 0.018 \\
1Au & 508 & 0.295 \\
2AuDHLA & 514 & 0.286 \\
3AuDHLANaCI & 514 & 0.267 \\
\hline 4AuLA & 514 & 0.290 \\
\hline 5AuLANaCI & 514 & 0.267 \\
\hline
\end{tabular}

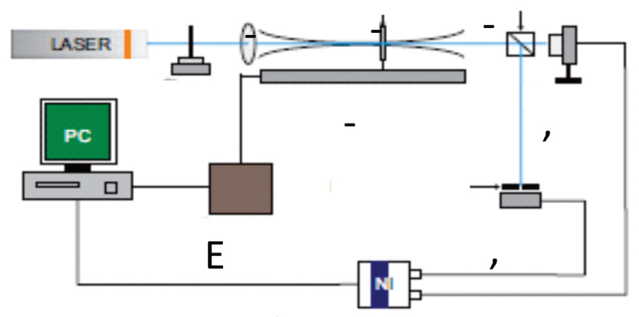

FIG. 2 Z-scan experimental setup.

the wave front phase, thereby modifying detected beam intensity. The nonlinear coefficient of refraction and absorption can be measured with a close and open aperture, respectively.

\section{RESULTS AND DISCUSSION}

A beam of laser at different power incised the solutions. The laser power was increased from $10 \mathrm{~mW}$ to $250 \mathrm{~mW}$, and rings were obtained by self-diffraction phenomena, except at 10 $\mathrm{mW}$ that they were not observed. At $25 \mathrm{~mW}$ it was possible to observe one ring, the self-diffraction phenomena, note that the number of the rings was minimum. It implies that we had a low phase shift. In Figure 3, it is shown the results for open aperture Z-scan measurement of the samples at different powers. It was not possible to determine the values of nonlinear absorption for all samples by simply using Eq. (??), because the adjustment of R-square was less than 0.7 in most of the samples, despite the tendency shown by the experimental data. Figure 3 shows a typical curve of two-photon absorption (TPA). The best adjustment was for the $5 \mathrm{AuLANaCl}$ sample, $\beta=10.7258 \times 10^{-6} \mathrm{~m} / \mathrm{W}$, and the 3AuDHLANaCl sample, $\beta=10.9258 \times 10^{-6} \mathrm{~m} / \mathrm{W}$ was obtained at $25 \mathrm{~mW}$ with $R^{2}=0.60939$ and $R^{2}=0.63395$, respectively. Nadjari et al. [44] report a $\beta=23 \times 10^{-6} \mathrm{~m} / \mathrm{W}$ for gold nanocolloids at $532 \mathrm{~nm}$ and $5.2 \times 10^{-6}$ fractional volume. Interestinlgy, despite that we had smaller Au nanoparticles concentration, the value order of $\beta$ was similar, because we were exciting at peak of plasmon wavelength.

We obtained the data using the PC-system for each solution; 

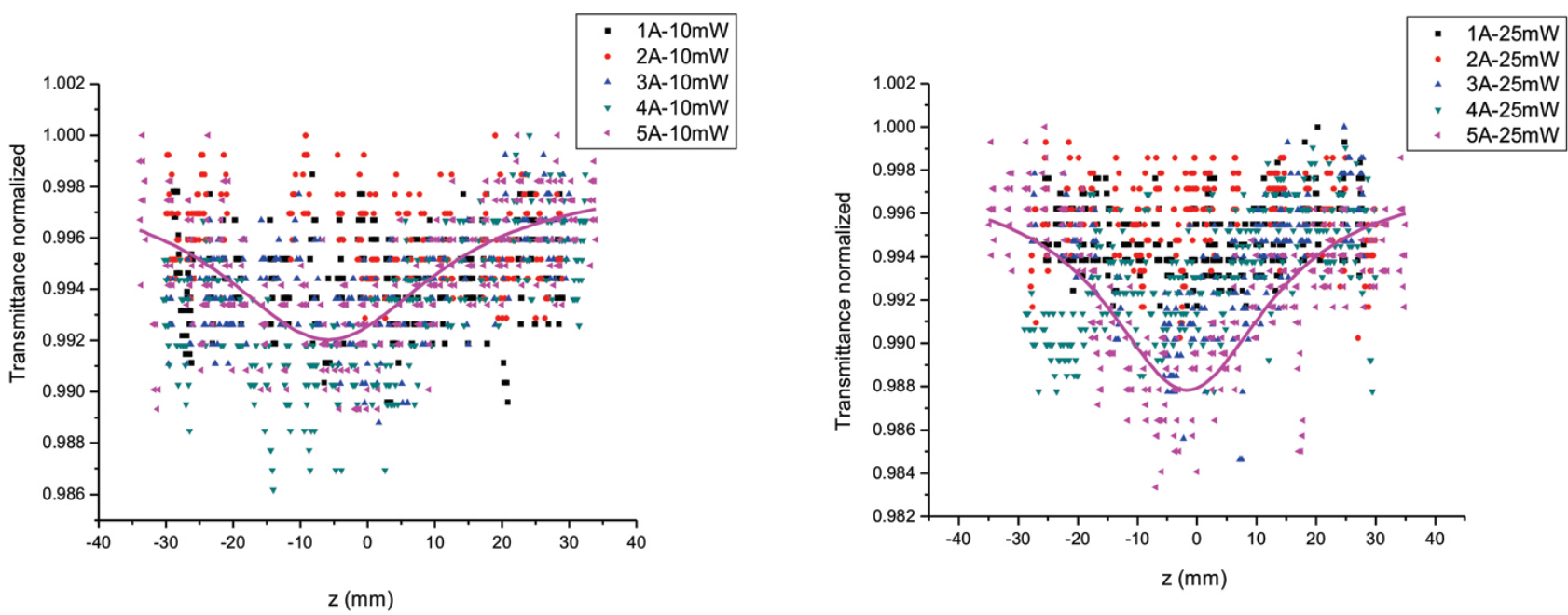

FIG. 3 Open aperture $z$-scan measurement to 5 solutions, $\beta=17 \times 10^{-6} \mathrm{~m} / \mathrm{W}\left(R^{2}=0.47\right)$ and $10.72 \times 10^{-6} \mathrm{~mW}\left(R^{2}=0.60\right)$ to $10 \mathrm{~mW}$ and $25 \mathrm{~mW}$ respectively were obtained using Eq. (6).

closed aperture $\mathrm{z}$-scan measurement to $1 \mathrm{Au}$ solution at different powers and frequencies were shown at Figure 4, the curves were fit using Eq. (3). Depending on the experimental conditions and on the sample absorption properties, thermal nonlinearities must be taken into account, especially on a time scale of milliseconds, the response given by Eq. (3) also describes systems nonlocal time, once they have reached a steady state [35]. Our experimental conditions allowed to apply laser wavelength to the colloid SPR peak to enhance the linear absorption, and this induced a larger thermal nonlinear refraction in the medium. It must be noticed that the SPR peak position was related to the gold nanoparticle size; therefore, this effect can play important roles on its thermo-optical properties. It is common that, in liquids, the local refractive index decreases with the increase of temperature, therefore, a temperature gradient result in a change of the local refractive index of the solution. In our case, we have an intense heat accumulated because the irradiation is at $514 \mathrm{~nm}$ with a CW laser, which corresponds to the resonance absorption peak of Au nanoparticles. This effect leads to the creation of larger thermal-induced negative nonlinear refractive index inside the solutions. Additionally, in this kind of thermal process, the nonlinear refraction index $\left(n_{2}\right)$ is not a constant value and depends on the incident intensity.

Figure 5 shows the behavior of parameters $\left(n_{2}, \theta, d n / d t\right)$ of samples according to the laser power. $\theta$ (thermal induced phase shift) and $d n / d t$ (thermal-optic coefficient) are not constant values, these depend on the incident laser intensity and the intrinsic properties of the material as $\kappa$, and $D$ (thermal conductivity and thermal diffusion respectively), because a radial direction was taking place in the diffusion of the heat (perpendicular to the $\mathrm{z}$ direction), then temperature field with spatial distribution had risen and had a different distribution to the laser beam temperature. As a consequence, the physical properties of the Au nanoparticles changed, affecting the propagation of the light beam itself. Note $\theta$, increases their value with the increase of the laser power due that the temperature was increased in consequence, self-diffraction occurred, while $d n / d t$, and $n_{2}$, which are negative, decreased. However,

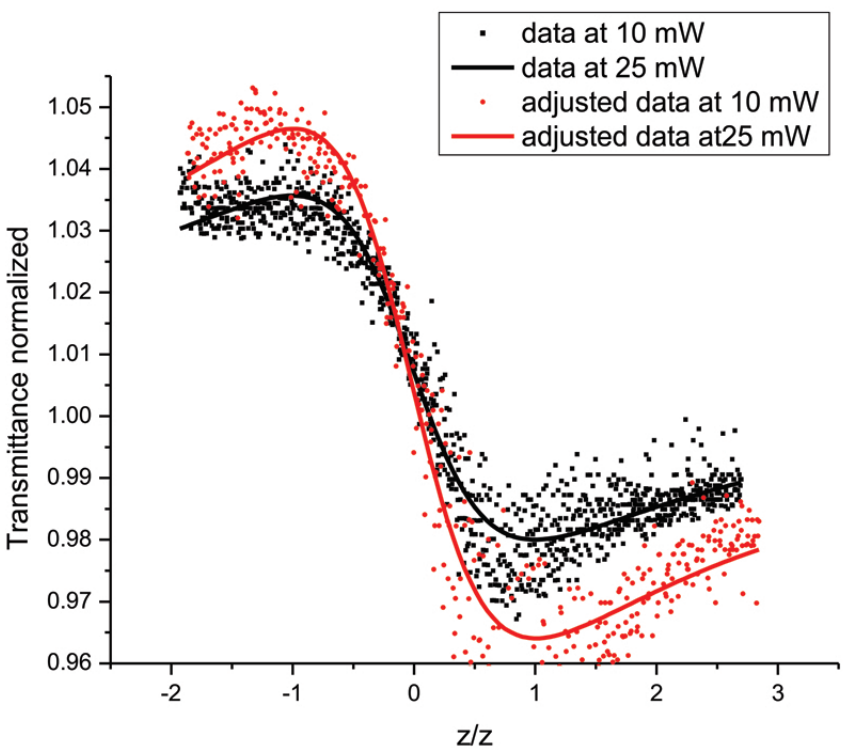

FIG. 4 Close aperture z-scan measurement to 1 Au solution at $100 \mathrm{hz}$ and $10 \mathrm{~mW}$ and $25 \mathrm{~m}$ using Eq. (3).

we note the thermal effect decreases with the frequency increase.

Table 2 shows values, standard error and adjusted R-square to each of $n_{2}, \theta, d n / d t$ and $\sigma$ were obtained for each solution at $10 \mathrm{~mW}$ and $100 \mathrm{~Hz}$ respectively. These values were obtained using Eq. (3), Eq. (9), Eq. (8) and Eq. (1), respectively. Commonly, the tendency of thermal conductivity enhancement with increase of nanoparticles concentration was observed. However, Shalkevich et al [?] makes reference to that the in tests done in fluids, only small changes in the thermal conductivity are within the uncertainty of the measurements. Accordingly we consider that due to the nanoparticles concentration being of $0.0004 \%$ per volume is low, and the size of the nanoparticles on average is of $4.5 \mathrm{~nm}$ and that the use of $\mathrm{k}$ value will be close to the reported in [?], thus, using Eq. (8), this shall imply that the $\mathrm{dn} / \mathrm{dt}$ value shall be the minimum ex- 

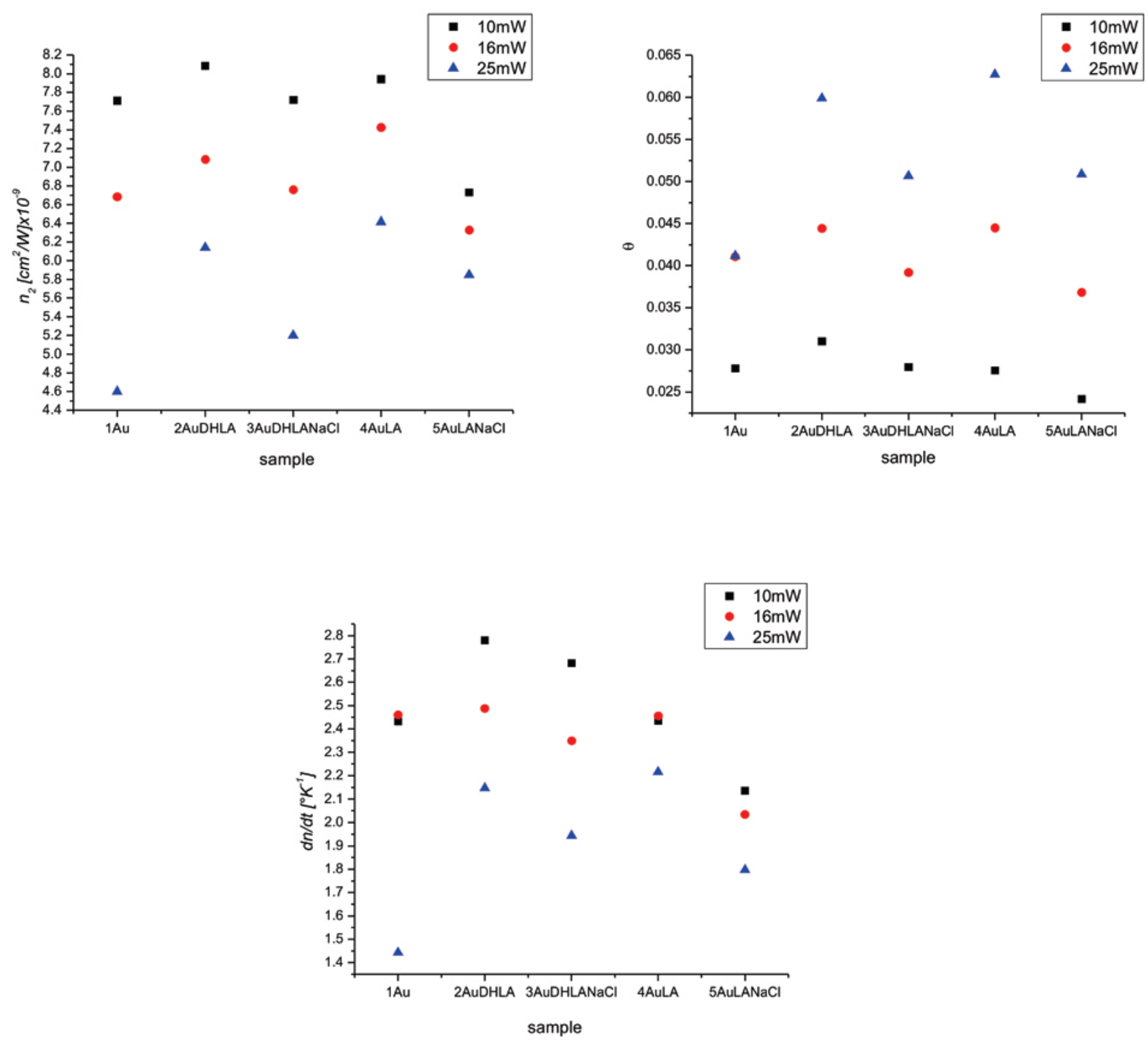

FIG. $5 n_{2}, \theta, d n / d t$, to different powers at $100 \mathrm{~Hz}$ to five samples.

pected. The sigma value is in agreement with the heating that firstly occurs close to the entering face, due to the strong absorption enriched by the SPR phenomena. Some simulations confirm this kind of process [35] which report having this behavior within their samples.

Analyzing the changes of five samples at $100 \mathrm{~Hz}$ and different powers, we noted that $n_{2}, \theta$, and $d n / d t$, how the presence of lipodic acid (reduced or not) increased the value of these parameters but when the $\mathrm{NaCl}$ was added it decreased the values, this was due to the fact that the $\mathrm{NaCl}$ solution has a lower
$C_{p}$ (specific heat), which decreased the thermal diffusion time, besides it is an ionic liquid, the ionic liquid has nonlinear phenomenon nonlocal too [45], producing a reduction in the selfdiffraction phenomenon (see Figure 5).

\section{CONCLUSIONS}

All Au solutions prepared presented nonlinear optical properties. The origin of these properties is thermal. Thermal nonlinear response is an important area for photonic applications

\begin{tabular}{|c|c|c|c|c|c|c|c|c|c|c|}
\hline sample & $\begin{array}{l}n_{2} \times 10^{-9} \\
{\left[\mathrm{~cm}^{2} / W\right]}\end{array}$ & $\begin{array}{c}\text { Standard } \\
\text { error } \\
{\left[\times 10^{-11}\right]}\end{array}$ & $\begin{array}{c}\text { Adj. } \\
\text { R-square }\end{array}$ & $\theta$ & $\begin{array}{c}\text { Standard } \\
\text { error } \\
{\left[\times 10^{-4}\right]}\end{array}$ & $\begin{array}{c}\text { Adj. } \\
\text { R-square }\end{array}$ & $\begin{array}{c}d n / d t \\
x 10^{-4} \\
{\left[{ }^{\circ} \mathrm{K}^{-1}\right]}\end{array}$ & $\begin{array}{c}\text { Standard } \\
\text { error } \\
{\left[\times 10^{-6}\right]}\end{array}$ & $\begin{array}{c}\text { Adj. } \\
\text { R-square }\end{array}$ & $\sigma$ \\
\hline $1 \mathrm{Au}$ & -7.70999 & 4.872668 & 0.96299 & 0.02781 & 1.70616 & 0.96432 & 1.55139 & 0.915943 & 0.96443 & 0.356183372 \\
\hline 2AuDHLA & -8.08399 & 5.16374 & 0.96124 & 0.03103 & 2.01313 & 0.96281 & 1.77325 & 1.09507 & 0.96292 & 0.345291527 \\
\hline 3AuDHLANaCl & -7.71928 & 4.65434 & 0.96809 & 0.02795 & 1.65631 & 0.96846 & 1.71094 & 1.00868 & 0.96850 & 0.35551376 \\
\hline 4AuLA & -7.9402 & 5.46494 & 0.95142 & 0.02756 & 2.15928 & 0.94913 & 1.5532 & 1.12223 & 0.94928 & 0.363114899 \\
\hline 5AuLANaCl & -6.7296 & 4.65399 & 0.95723 & 0.02417 & 1.72777 & 0.95322 & 1.47409 & 1.0005 & 0.95336 & 0.357616899 \\
\hline
\end{tabular}


that require $\mathrm{CW}$ or higher power lasers, where thermo optical effects can be exploited to be able to use nonlocal nolinear phenomena, such as spatial solitons and shock waves. Au nanoparticles properties depend on wavelength and intensity laser. Presence of lipodic acid increase the $n_{2}, \theta$ and $d n / d t$ values. Presence of $\mathrm{NaCl}$ decreases these values, this implies we can controlled the nonlocal linearity and add or substract $\mathrm{NaCl}$ solution or ionic liquids at samples.

\section{References}

[1] U. Kreibing, and M. Vollmer, Optical properties of metal clusters (Springer, Berlin, 1995).

[2] P. Mulvaney, "Surface Plasmon Spectroscopy of Nanosized Metal. Particles," Langmuir 12, 788-800 (1996).

[3] S.-Z. Tan, Y.-J. Hu, J.-W. Chen, G.-L. Shen, and R.-Q. Yu, "An optical sensor based on covalent immobilization of 1-aminopyrene using Au nanoparticles as bridges and carriers," Sensor. Actuat. B-Chem 124(1), 68-73 (2007).

[4] D. Zheng, C. Hu, T. Gan, X. Dang, and S. Hu, “Preparation and application of a novel vanillin sensor based on biosynthesis of Au-Ag alloy nanoparticles," Sensor. Actuat. B-Chem 14(1), 247-252 (2010).

[5] F. Lu, T. L. Doane, J.-J. Zhu, C. Burda, "2Cold nanoparticles for diagnostic sensing and therapy," Special Issue: Metals in Medicine, Inorganica Chimica Acta 393, 142-153 (2012).

[6] W. B. Liechty, and N. A. Peppas, "Expert opinion: Responsive polymer nanoparticles in cancer therapy," Eur. J. Pharm. Biopharm. 80(2), 241-246 (2012).

[7] P. K. Jain, I. H. El-Sayed, M. A. El-Sayed, "Au nanoparticles target cancer," Nano Today 2(1), 18-29 (2007).

[8] M. E. Greene, "Nanoparticles help paint resist germs: Nanotechnology," Mater. Today 11(3), 16 (2008).

[9] A. A. Dakhel, "Preparation and optical study of Au nanograins in amorphous La-oxide medium," Colloid. Surface. A 332, 9-12 (2009).

[10] N. Shalkevich, W. Escher, T. Bürgi, B. Michel, L. Si-Ahmed, and Dimos Poulikakos, "On the Thermal Conductivity of Cold Nanoparticle Colloids," Langmuir 26(2), 663-670 (2010).

[11] S. A. Putnam, D. G. Cahill, P. V. Braun, Z. Ge, and R. G. Shimmin, "Thermal conductivity of nanoparticle suspensions," J. Appl. Phys. 99, 084308 (2006).

[12] H. E. Patel, S. K. Das, T. Sundararajan, A. Sreekumaran Nair, B. Ceorge, and T. Pradeep, "Thermal conductivities of naked and monolayer protected metal nanoparticle based nanofluids: Manifestation of anomalous enhancement and chemical effects," Appl. Phys. Lett. 83, 2931 (2003).

[13] D. Lee, "Thermophysical properties of interfacial layer in nanofluids," Langmuir 23(11), 6011-6018 (2007).

[14] S. U. S. Choi, Z. G. Zhang, W. Yu, F. E. Lockwood, and E. A. Grulke, "Anomalous thermal conductivity enhancement in nanotube suspensions," Appl. Phys. Lett. 79, 2252 (2001).

[15] H. Yang, L. Zhang, and X. Fu, "Spectral evolution of an optical pattern generated by spatial modulation instability in a reorientational Kerr nonlinear medium," J. Mod. Optic. 59(2), (2012).

[16] R. E. Noskov, P. A. Belov, and Y. S. Kivshar, "Subwavelength Modulational Instability and Plasmon Oscillons in Nanoparticle Arrays," Phys. Rev. Lett. 108, 093901 (2012).
[17] B. Gu, F. Ye, K. Lou, Y. Li, J. Chen, and H.-T. Wang, "Vectorial selfdiffraction effect in optically Kerr medium," Opt. Express 20(1), 149 (2012).

[18] B. V. Ana, and K. Saravanamuttu, "Diversity and slow dynamics of diffraction rings:a comprehensive study of spatial self-phase modulation in a photopolymer," J. Opt. Soc. Am. B 29(8), 23572372 (2012).

[19] W. J. Wan, S. Jia, "Dispersive superfluid-like shock waves in nonlinear optics," Nat. Phys. 3, 46 (2007).

[20] N. Ghofraniha, C. Conti, G. Ruocco, and S. Trillo, "Shocks in nonlocal media," Phys. Rev. Lett. 99, 043903 (2007).

[21] A. I. Yakimenko, Y. A. Zaliznyak, and Y. S. Kivshar, "Stable vortex solitons in nonlocal self-focusing nonlinear media," Phys. Rev. E 71, 065603(R) (2005).

[22] B. Alfassi, C. Rotschild, and M. Segev, "Incoherent surface solitons in effectively instantaneous nonlocal nonlinear media," Phys. Rev. A 80, 041808 (2009).

[23] S. Namiki, T. Kurosu, K. Tanizawa, S. Petit, G. Mingyi, and J. Kurumida, "Controlling Optical Signals Through Parametric Processes," IEEE J. Sel. Top. Quant. 18(2), 717-725 (2012).

[24] X. Q. Wang, Q. Ren, F. J. Zhang, W. F. Guo, X. B. Sun, J. Sun, H. L. Yang, et al., "Preparation, characterization, thermal and thirdorder nonlinear optical properties of bis(tetraethylammonium) bis(2-thiox0-1,3-dithiole-4,5-dithiolato)cuprate(II)," Mater. Res. Bull. 43(8), 2342-2353 (2008).

[25] A. Y. Fasasi, M. Maaza, C. Theron, et al., "Non-linear absorption and second harmonic imaging of $\mathrm{Zn}-\mathrm{BaTi0} 3$ thin films prepared by laser ablation," Thin Solid Films 516(18), 6233 (2008).

[26] Y. H. Wang, J. D. Lu, R. W. Wang, S. J. Peng, Y. L. Mao, and Y. G. Cheng, "Optical nonlinearities of Au nanocluster composite fabricated by $300 \mathrm{keV}$ ion implantation," Physica B. 403, 3399-3402 (2008).

[27] Y. H. Wang, Y. M. Wang, J. D. Lu, L. L. Ji, R. G. Zhang, and R. W. Wang, "Nonlinear optical properties of Cu nanoclusters by ion implantation in silicate glass," Opt. Commun. 283, 486-489 (2010).

[28] X.-X. Yu, and Y.-H. Wang, "Measurement of nonlinear optical refraction of composite material based on sapphire with silver by Kerrlens autocorrelation method," Opt. Express 22, 177-18 (2014).

[29] S. Qu, C. Zhao, X. Jiang, G. Fang, Y. Gao, H. Zeng, Y. Song, et al., "Optical nonlinearities of space selectively precipitated $\mathrm{Au}$ nanoparticles inside glasses," Chem. Phys. Lett. 368, 352-358 (2003).

[30] S. Qu, Y. Zhang, H. Li, J. Qiu, and C. Zhu, "Nanosecond nonlinear absorption in $\mathrm{Au}$ and $\mathrm{Ag}$ nanoparticles precipitated glasses induced by femtosecond laser," Opt. Mater. 28, 259-265 (2006).

[31] P. Lesiak, and M. Wójcik, "Nonlinear optical characterization of the gold nanoparticles coated by thiols," Photonics Letters of Poland 3(3), 113-115 (2001).

[32] H. I. Elim, J. Yang, J.-Y. Lee, J. Mi, and Wei Ji, "Observation of saturable and reverse-saturable absorption at longitudinal surface Plasmon resonance in gold nanorods," Appl. Phys. Lett. 88, 083107 (2006).

[33] Y. Zhang, M. Ma, X. Wang, D. Fu, N. Gu, et al., "Second-order optical nonlinearity of surface-capped CdS nanoparticles and effect of surface modification," J. Phys. Chem. Solids 64, 927-931 (2003).

[34] N. Ghofraniha, C.Conti, G. Ruocco, and S.Trillo, "Shocks in Nonlocal Media," Phys. Rev. Lett. 99, 043903 (2007). 
[35] M. Sheik-Bahae, A. A. Said, T. H. Wei, D. J. Hagan, and E. W. VanStryland, "Sensitive measurement of optical nonlinearities using a single beam," IEEE J. Quant. Electron 26, 760 (1990).

[36] J. P Gordon, R. C. C. Leite, R. S. Moore, S. P. S. Porto, and J. R. Whinnery, "Long transient effects in laser with Inserted liquid samples", J. Appl. Phys. 36(1), 3-8 (1965) .

[37] C. A. Carter, and J. M. Harris, "Comparasion of models describing the thermal lens effect," Appl. Optics 23, 476-481 (1984).

[38] E. W. Van Stryland, and M. Sheik-Bahae "Z-Scan Measurements of Optical Nonlinearities," in Characterization Techniques and Tabulations for Organic Nonlinear Materials M. G. Kuzyk, and C. W. Dirk, Eds., 655-692 (Marcel Dekker, Inc., New York City, 1998).

[39] S. A. Akhmanov, D. P. Krindach, A. V. Migulin, A. P. Sukhroukov, and R. V. Khokhlov, "Thermal self-actions of laser beams," IEEE J. Quant. Electron. 4(10), 568-575 (1968).

[40] C. A. Carter, and J. M. Harris, "Comparison of models describing the thermal lens effect," Appl. Optics 23, 476-481 (1984).
[41] M. Brust, M. Walker, D. Bethell, D. J. Schiffrin, and R. Whyman, "Synthesis of thiol-derivatised nanoparticles in two-phase liquidliquid system," J. Chem. Soc., Chem. Commun. 7, 801-802 (1994).

[42] W. Haiss, N. T. K. Thanh, J. Aveyard, and D. G. Fernig, "Determination of size and concentration of gold nanoparticles from UV-Vis spectra," Anal. Chem. 79, 4215-4221 (2007).

[43] C. C. López-Mora, M. Trejo-Duran, E. Alvarado-Méndez, R. RojasLaguna, E. Vargas-Rodríguez, J. M. Estudillo-Ayala, R. Mata-Chavez, et al., "PC-Based systems for experiments in optical characterization of materials," J. Phys. Conf. Ser. 274, 12059 (2011).

[44] H. Nadjari, F. Hajiesmaeilbaigi, and A. Motamedi, "Thermo Optical Response and Optical Limiting in $\mathrm{Ag}$ and Au Nanocolloid Prepared by Laser Ablation," Laser Phys. 20(4), 859-864 (2010).

[45] M. Trejo-Duran, E. Alvarado-Mendez, E. Vargas-Rodriguez, J. M. Estudillo-Ayala, and R. I. Mata-Chavez, "Nonlinear optical characterization of ionics liquids of 1-methylpyrrolidine family," Proc. SPIE 8412, 84121X-1 (2012). 